\title{
LA PNEUMATOlOGie DE VATICAN II
}

\author{
The Pneumatology of the Second Vatican Council
}

Gilles Routhier *

RÉSUMÉ: La pneumatologie du Concile Vatican II figurait parmi les Questiones disputatae entre théologiens orthodoxes, protestants et catholiques après le Concile. Cette génération ayant disparu, il est important de dresser un nouveau bilan. Pour ce faire, il ne suffit pas de compter le nombre d'occurrences du terme Saint-Esprit dans le texte conciliaire, mais il importe de voir si le recours au Saint-Esprit détermine la perspective théologique adoptée par le Concile et intervient à des points stratégiques de l'argumentation. Un nouvel examen de la question conduit à l'identification de trois domaines où la pneumatologie conciliaire joue un rôle important en ecclésiologie : l'Esprit Saint est co-instituant de l'Église. Il est en même temps source de la diversité et de l'unité, si bien qu'on ne peut la concevoir que comme unité catholique; enfin, le fait qu'il dispense ses dons à tous les fidèles fonde la participation de tous à la vie de l'Église. De plus, non seulement l'Esprit habite dans l'Église comme en un temple, mais il remplit aussi l'univers, précédant l'action de l'Église et en dépassant les frontières. En conclusion, l'essai souligne le fait que les développements postconciliaires sur des questions cruciales, telles que la théologie des ministères, la réflexion sur le sensus fidei et la synodalité, dépendent des fondements posés par Vatican II. De plus, la théologie de la mission et la théologie des religions n'auraient pas connu de tels développements sans la réflexion conciliaire sur le rôle du Saint-Esprit dans le monde et au-delà des frontières de l'Église.

MOTS-CLÉS: Saint-Esprit. Concile Vatican II. Pneumatologie. Ecclésiologie. Lumen Gentium.

SUMMARY: The pneumatology of the Second Vatican Council was among the Questiones disputatae between Orthodox, Protestants and Catholics theologians after the Council. Now that this generation has faded, it is important to draw up a new

* Université Laval, Québec, Canadá. 
balance sheet. To do this, it is not enough to count the number of occurrences of the term Holy Spirit in the conciliar text, but to see if the recourse to the Holy Spirit determines the theological perspective adopted by the Council and intervenes at strategic points in the argumentation. A new examination of the question leads to the identification of three areas where conciliar pneumatology plays an important role in ecclesiology: the Holy Spirit is The Holy Spirit co-established the Church; He promotes at the same time unity and diversity, so that unity can only be conceived as "Catholic unity". Finally, His gifts, given to all the baptized, founds the participation of all the faithful in the life of the Church. Not only the Holy Spirit dwells in the Church as in His Temple, but He fills the universe and precedes and surpasses the action of the Church. In conclusion, this essay underlines the fact that post conciliar developments on crucial questions, such as the theology of ministries, reflection on the sensus fidei and synodality are dependent on the foundations laid by Vatican II. Furthermore, the theology of mission and the theology of religions would not have known such developments without the conciliar reflection on the role of the Holy Spirit in the world and beyond the borders of the Church.

KEYWORD: Holy Spirit. Second Vatican Council. Pneumatology. Ecclesiology. Lumen Gentium.

L es conciles, « légitimement réunis dans l'Esprit Saint » (Trente, session IIII) sont conçus comme des événements pneumatiques et se sont eux-mêmes perçus comme nouvelle pentecôte. Déjà Athanase avait qualifié le premier concile de Nicée de "Nouvelle pentecôte » (ATHANASE, Apologia secunda 7, 2), expression reprise à plusieurs reprises par le pape Jean XXIII pour qualifier le concile Vatican II. En effet, désireux de partager l'intuition spirituelle profonde qu'il portait au moment de convoquer un concile, Jean XXIII procédait en utilisant diverses images. Il évoquait le printemps, le concile apparaissant comme " une fleur d'un printemps inattendu »(Motu proprio Superno Dei nutu, 5 juin 1960), ou un vent d'air frais qui venait rafraîchir l'Église, image qui n'était pas sans évoquer l'action de 1'Esprit Saint ${ }^{1}$, mais il a eu principalement recours à l'image de la Pentecôte. De plus, il ne manqua pas de célébrer de manière spéciale les fêtes de la Pentecôte qui ont jalonné la préparation du concile ${ }^{2}$. Cette image, qu'il utilise pour la première fois le 27 mai 1959, sera récurrente

\footnotetext{
${ }^{1}$ Suivant le témoignage du journaliste $H$. Fesquet, à un visiteur qui lui demandait ce qu'il attendait du Concile, Jean XXIII, en montrant la fenêtre, aurait fait la réponse suivante: « un courant d'air frais dans l'Église » (FESQUET, 1966, p. 49).

${ }^{2}$ Ainsi, il met sur pied la commission antepréparatoire lors de la Pentecôte 1959 (le 17 mai - Acta et documenta Concilio oecumenico Vaticano II apparando [AD]. Series I (Antepreparatoria): Acta S. Pontificis Ioannis XXIII. Vatican: Typis polyglottis vaticani, 1960. v. 1. p. 24) ; procède à l'ouverture de la phase préparatoire en la fête de la Pentecôte en 1960 (le 5 juin - Id., p. 105). Pour marquer la Pentecôte de 1961, il publie sa lettre apostolique Celebrandi concilii Oecumenici (11 avril - AD, Series II (Praeparatoria), vol. I, p. 76).
} 
dans ses interventions ${ }^{3}$. Elle revient avec force dans son allocution de clôture de la première session (8 décembre 1962) où il évoque « la nouvelle Pentecôte si attendue » :

Ce sera alors vraiment la nouvelle Pentecôte si attendue, qui enrichira l'Église de nouvelles forces spirituelles et fera rayonner davantage son esprit maternel et son action salutaire dans tous les domaines de l'activité humaine. Ce sera un nouveau bond en avant du royaume du Christ dans le monde, une nouvelle proclamation, toujours plus profonde et plus persuasive, de la joyeuse nouvelle de la Rédemption (Acta Synodalia [AS], I/IV, 391-393)4.

Événement pneumatique, les conciles sont inaugurés par une invocation à l'Esprit et c'est sous la conduite du même Esprit, dont l'activité se manifeste dans la concorde, que les Pères mènent leurs travaux. Toutefois, affirmer la place éminente de l'Esprit Saint dans le dessein du concile d'Angelo Roncalli ne suffit pas pour affirmer que le concile lui-même a donné une place importante à l'Esprit Saint dans son enseignement. C'est cet enseignement que l'on voudra scruter dans le présent article, m'attachant au texte de Vatican II, laissant de côté la question de la réception de cette pneumatologie, même si cette question mériterait une autre étude.

Au cours de la décennie qui a suivi Vatican II, le débat a été vif quant à l'importance accordé à l'Esprit Saint dans les textes conciliaires eux-mêmes. Ainsi, la pneumatologie du concile Vatican II a souvent été jugée déficiente non seulement par les orthodoxes ${ }^{5}$ et les protestants ${ }^{6}$. Ces critiques, souvent nuancées ${ }^{7}$, ont été reprises par leurs interlocuteurs catholiques qui ont répondu avec précision à ces critiques ${ }^{8}$. Dans

${ }^{3} \mathrm{Il}$ compare à nouveau le concile à une nouvelle pentecôte dans la prière à l'Esprit Saint qu'il propose pour le concile à l'été 1959 (Ibid., p. 59). Le motif de la nouvelle pentecôte est repris dans une lettre qu'il adresse au cardinal Alfrink (28 mai 1960 - Ibid., p. 87).

${ }^{4}$ Traduction de La Documentation catholique, T. LX, n. 1391, p. 11, 1963.

${ }^{5}$ Pour un point de vue orthodoxe, on verra Nissiotis (1965, en particulier la section 3: "An Ecclesiology without Pneumatology", p. 48-61; 1966, p. 249-252; 1967, p. 324-325 : ici l'auteur reconnaît la mention de l'Esprit en Lumen Gentium, mais il y est subordonné au Christ) et Clément (1966). Ces remarques sont tempérées notamment par un éminent théologien orthodoxe, Jean Zizioulas, soucieux d'opérer une synthèse entre pneumatologie et christologie (ZIZIOULAS, 1981).

${ }^{6}$ Pour un point de vue protestant, cf. Roux (1967, p. 112-114); Vajta (1965, p. 107); Vischer (1964, p. 45); Westphal (1966, p. 134). Selon ce dernier, on n'aurait que «saupoudré» de la pneumatologie en Lumen Gentium.

"Par exemple, sous le titre "An Ecclesiology without Pneumatology", Nissiotis commence par écrire : "Now however, after the severe criticism of the Fathers of the Council and of the observers, the new revised Schema De Ecclesia mentions the Holy Spirit at four crucial places in the text, and, what is most encouraging, the event of Pentecost as contributing to the realisation of the firs community of believers. This is a very positive sign [...]» (NISSIOTIS, 1965, p. 49). Dans son rapport au COE, il écrit : "Sur ce point pourtant, de grands progrès ont été faits pendant les quatre ans qu'a duré le Concile » (NISSIOTIS, 1966, p. 249).

${ }^{8}$ G. Philips, principal rédacteur de Lumen Gentium, a relevé ces critiques. Après avoir évoqué la place faite à l'Esprit dans cette Constitution, il conclut : " Comment prétendre encore que le Concile n'attache que peu ou même pas d'importance à la pneumatologie » (PHILIPS, 
ce concert, l'opinion de Louis Bouyer se détache par son caractère unilatéral et sans nuance:

On est frappé par-dessus tout de deux lacunes générales de l'enseignement de Lumen gentium, pour lesquelles on chercherait vainement dans les autres textes conciliaires quelque compensation. Ces deux manques peuvent apparaître comme antithétiques. Mais leur simultanéité n'en est que plus frappante. La Constitution sur l'Église ignore à peu près complètement le droit canon... Mais, chose curieuse, à part un beau paragraphe, plus pieux que doctrinal, elle n'ignore guère moins complètement le Saint-Esprit! (BOUYER, 1970, p. 208-209)

C'est sans doute Y. Congar qui tentera, nous le verrons abondamment dans cet article, d'entrer en dialogue et de nuancer cette critique adressée, tout en souhaitant en tirer profit:

Les Observateurs au deuxième concile du Vatican ont souvent reproché aux schémas, en particulier à ceux des constitutions dogmatiques Lumen gentium et Dei verbum, de manquer de pneumatologie. L'un d'eux, Nikos A. Nissiotis, [...], articule le même reproche; il est revenu avec insistance sur ce qu'il appelle le « christomonisme » des Latins et une « pneumatologie ecclésiologique » dont les Latins manquent et qui serait l'âme de l'ecclésiologie Orthodoxe.

Les Latins tendent à faire du Saint-Esprit une simple fonction du Christ : fonction de personnaliser le salut (ce qu'on appelle parfois improprement la " rédemption subjective »), fonction d'assurer l'harmonie de la vie ecclésiale, son développement, sa fidélité à ses origines par les charismes institutionnels ou personnels ; bref, fonction d'effectuer, dans l'Église, l'œuvre $d u$ Christ : Tertullien n'appelle-t-il pas le Saint-Esprit « vicarius Christi » (Præscr., c. 28 ; comp. C. 13) ? C'est méconnaître le caractère pleinement personnel de la mission du Saint-Esprit à la Pentecôte, sa valeur propre pour constituer l'Église après et avec l'œuvre christologique, enfin l'action personnelle de la troisième Hypostase dans la vie historique de l'Église. (CONGAR, 1970, p. 41-42)

Toutes les opinions que nous venons de passer en revue sont le fait d'acteurs du concile, soit à titre d'experts au concile ou de peritus privatus, soit à titre d'observateurs non-catholiques. Comment peut-on aujourd'hui, à distance et alors que cette génération disparaît de notre mémoire, apprécier la pneumatologie de Vatican II ? Je privilégierai ici deux approches, tout en en excluant une troisième. Je n'emprunterai pas ici la voie statistique qui consisterait à compter le nombre d'occurrences des expressions « Esprit Saint » ou équivalentes dans le corpus textuel de Vatican II et de comparer ce résultat avec ce que l'on obtient dans les corpus des autres conciles. Cette méthode purement

1967, T. I, p. 232; T II, p. 32-33). Y. Congar s'est aussi montré sensible à ces critiques et leur a opposé une démonstration qui en réduit au moins la portée (CONGAR, 1981, p. 117-130; CONGAR, 1979, T. I, specialment le chapitre X «La pneumatologie de Vatican II», p. 227-235). On pourrait remonter premier article de Congar à la suite de Vatican II sur la pneumatologie dans la théologie catholique (CONGAR, 1967). Il est essentiellement construit à partir de la critique de Nissiotis. On lira aussi l'importante contribution de Charrue (1969) qui relate l'introduction de la pneumatologie dans le schéma De Ecclesia tout au long de sa rédaction (CAZELLE, 1968). 
statistique, Congar en avait déjà indiqué le potentiel, mais également les limites (voir CONGAR, 1984, p. 73-78). D'une part, les corpus ne sont pas de volume équivalent, ce qui rend la comparaison impossible. D'autre part, même si le résultat pour Vatican II est inégalé et impressionnant ${ }^{9}$, la seule statistique ne serait pas convaincante. En effet, on pourrait toujours dire qu'on n'a fait que saupoudrer cette mention dans les textes, sans qu'elle ne soit déterminante. Une comptabilité sèche des termes utilisés commande donc d'être complétée par au moins deux autres approches : d'une part, identifier le développement de la pneumatologie de Vatican II en s'intéressant à l'iter de la rédaction de ses textes. D'autre part, examiner si les mentions de l'Esprit Saint se situent à des lieux stratégiques et déterminent la pensée plutôt que de simplement orner les développements sur une question.

Ce faisant, je tenterai, modestement, de dresser un status quaestionis sur la place de l'Esprit Saint dans les textes de Vatican II, cette question ayant déjà fait l'objet de plusieurs travau ${ }^{10}$, comme la bibliographie offerte en notes permet de le constater. Cela pourra également répondre au vœu de Paul VI qui indiquait que «à la christologie et spécialement à l'ecclésiologie du Concile doivent succéder une étude nouvelle et un culte nouveau de l'Esprit-Saint, précisément comme complément indispensable de l'enseignement du Concile » (Audience générale, 6 juin 1973).

\section{Une place de plus en plus importante accordée à l'Esprit Saint dans l'ecclésiologie conciliaire}

La comparaison des différents états rédactionnels des textes conciliaires nous conduit à une conclusion : plus le concile évolue, plus on accorde de la place à l'Esprit Saint dans la théologie conciliaire. À défaut de pouvoir reprendre ici l'iter rédactionnel des seize textes de Vatican II, je me limiterai au De Ecclesia. Il aurait sans doute fallu élargir l'enquête, mais ce texte est déjà révélateur de la place réservée à l'Esprit Saint dans la théologie de Vatican II, le credo Ecclesiam étant étroitement rattaché au credo in Spiritum Sanctum dans la confession de foi.

La simple lecture synoptique des différents schémas qui ont conduit au texte final de Lumen Gentium ${ }^{11}$ nous montre à l'évidence que, de marginal

\footnotetext{
${ }^{9}$ Il y a 410 mentions de l'Esprit dans les textes de Vatican II, contre un total de 782 occurrences dans l'ensemble de 21 concile œcuméniques. C'est dire que les textes de Vatican II à eux seuls en compte plus de la moitié du total. Cela est déjà parlant. Voir CETEDOC. Theaurus conciliorum oecumenicorum et generalium Ecclesiae catholicae. Turnhout: Brepols, 1996. ${ }^{10}$ Parmi les travaux plus récents, je renvoie en particulier à Nisius (2006).

${ }^{11}$ Pour Lumen gentium, voir Alberigo et Magistretti (1975) et Gil Hellín (1995). Pour le chapitre VIII de la Constitution, voir Toniolio (2004). Pour le décret Presbyterorum ordinis, voir Wasselynck (1968) et Gil Hellín (1996).
} 
qu'il était dans les premières versions, le rôle de l'Esprit gagne progressivement du terrain dans les versions successives. Cela est particulièrement manifeste par l'ajout, en février 1963, d'un numéro complet consacré à l'Esprit Saint [De Spiritu Ecclesiam sanctificante]. Ce $\mathrm{n}^{\circ} 4$, absent des deux premiers schémas ${ }^{12}$ constitue sans doute l'innovation la plus importante et celle qui aura le plus de conséquence pour la suite.

La présentation de l'Église comme Temple de l'Esprit ${ }^{13}$, introduite dans la nouvelle version du texte en septembre 1963, s'est trouvée soutenue et confortée lors de la discussion du nouveau texte au cours de la deuxième session. On voulut même accroître cet accent pneumatologique du nouveau schéma ${ }^{14}$. Si le cardinal Confalonieri rappela l'importance de la Pentecôte lorsque l'on veut penser l'Église ${ }^{15}$, le cardinal Suenens et Mgr Ziadé, qui intervinrent sur le chapitre II (le peuple de Dieu) insistèrent sur la dimension charismatique de l'Église. Ce dernier arguait que, «selon la Tradition de l'Écriture et la tradition constante de l'Église d'Orient, il n'y a pas de théologie de l'Église qui ne soit pas pneumatologique, c'est-à-dire que l'Église est le mystère de l'effusion de l'Esprit aux derniers temps » (CONGAR; KÜNG; O'HANLON, 1964, p. 37). Ces interventions (sur le chapitre deux du schéma) ont eu une véritable influence sur l'évolution du texte. Non seulement le texte final du chapitre deux contient 23 références à l'Esprit Saint - sur les 110 que compte le schéma - mais la dimension charismatique de l'Église a été plus nettement mise en valeur ${ }^{16}$. De plus, l'affirmation de principe selon laquelle les dons de l'Esprit sont répandus sur tous les fidèles du Christ ${ }^{17}$ devait avoir des conséquences dans les chapitres III (sur les ministères ordonnés), IV (sur les laïcs ${ }^{18}$ ), V (sur l'appel de tous à la sainteté ${ }^{19}$ ) et VI (sur la vie religieuse ${ }^{20}$ ). Bien plus, cette pers-

\footnotetext{
${ }^{12}$ Schema constitutionis dogmaticae de Eccledia (nov. 1962) et Schema constitutionis De Ecclesia (nov. 1962).

${ }^{13}$ Cette façon de considérer l'Église est présente aux $n^{\text {os }} 4$ et 6 (passages introduits soit en février ou en avril 1963) et 9 et 17 (passages introduits au printemps et à l'automne 1964, après la discussion in aula à la deuxième session).

${ }^{14}$ Je pense en particulier aux interventions du cardinal Confalonieri (AS II/II, p. 16), à celle du card. Suenens (AS II/III, p. 175) et à celle de l'archevêque maronite de Beyrouth, Mgr Ziade (AS II/III, p. 211). Ces deux derniers textes ont été édités en français dans Congar, Küng et O'hanlon (1964, p. 31-42).

${ }^{15}$ Le texte final comporte deux références à la Pentecôte, aux ${ }^{\text {os }} 4$ et 24 , mentions ajoutées en 1963, et une autre au no 19, ajoutée en 1964.

16 On verra en particulier les $\mathrm{n}^{\text {os }} 9$ à 12 , en particulier le $\mathrm{n}^{\circ} 12$ sur «le sens de la foi et les charismes dans le peuple chrétien ».

17 « L'Esprit-Saint non seulement sanctifie le Peuple de Dieu, le conduit et l'orne de vertus au moyen des sacrements et des ministères mais, «en distribuant à chacun ses dons comme il lui plaît» (I Cor. 12, 11), il dispense également, parmi les fidèles de tout ordre, des grâces spéciales qui les habilitent à assumer des activités et des services divers, utiles au renouvellement et à l'expansion de l'Église, suivant ces paroles: «À chacun la manifestation de l'Esprit est donnée en vue du bien commun» (I Cor. 12, 7) ». (LG, n. 12)

${ }^{18}$ On verra en particulier les $\mathrm{n}^{\text {os }} 30,32$ et 34 .

${ }^{19}$ Voir en particulier les $n^{\text {os }} 39,40$ et 42 .

${ }^{20}$ Voir en particulier le $\mathrm{n}^{\circ} 44$.
} 
pective passera ensuite dans d'autres documents, notamment Presbyterorum ordinis, Perfectae caritatis et Apostolicam actuositatem, documents que nous n'analyserons pas de manière approfondie.

À l'évidence, le processus conciliaire qui a permis la maturation du texte a singulièrement infléchi le texte, si bien que, dans sa version finale, le texte attribue un rôle important à l'Esprit Saint.

\section{Des mentions de l'Esprit dans des lieux stratégiques de la constitution Lumen Gentium}

À l'examen, il apparaît que la référence à l'Esprit Saint, qui habite dans l'Église comme en son temple, joue deux rôles fondamentaux dans Lumen Gentium. Il est à l'origine et au fondement de la constitution et du maintien de l'Église, dans la sainteté, la vérité et l'unité. De la même manière, il est à l'origine et au fondement de l'activité apostolique de l'Église et de ses membres (ministres ordonnés et christi fideles laici) qui, ensemble, sont les bénéficiaires des dons variés de l'Esprit. Enfin, c'est l'onction du Saint qui donne à tous les fidèles cet instinct spirituel qui leur permet de discerner la vraie foi. C'est dire que cette référence est véritablement structurante et pas simplement décorative. Je considérerai successivement ces trois domaines où la référence à l'Esprit Saint joue un rôle déterminant.

\subsection{L'Esprit co-instituant de l'Église}

En premier lieu, il faut affirmer que Lumen Gentium prend ses distances par rapport à une ecclésiologie qui se limitait à présenter le Christ comme fondateur de l'Église, ce qui avait pour conséquence de ne concevoir l'Église qu'à partir de sa référence christologique. Vatican II opère un déplacement qui le conduit à développer une théologie des ministères qui s'éloigne du christomonisme qui l'inspirait (voir CONGAR, 1970). Lumen Gentium s'attache à présenter le surgissement de l'Église dans l'histoire comme événement lié à l'événement de la Pentecôte (cf. LG, n. 2, 4, 5, 13, etc.) et rattaché à l'Esprit Saint. Bien plus, son existence dans le temps est dépendante de $l^{\prime}$ Esprit qui la vivifie et la renouvelle (LG, n. 4, 7, 8, 9, etc.). On sort donc d'une conception de l'Église liée uniquement au Christ comme à son fondateur ou conçue comme l'incarnation continuée, perspective commune enseignée tout au long du XIX $X^{e}$ siècle. L'Église est située dans l'économie du salut et dans le déploiement trinitaire de cette économie ${ }^{21}$.

\footnotetext{
${ }^{21}$ Cette perspective est développée dans des lieux stratégiques : le prologue trinitaire de Lumen Gentium, nos 2 (le dessein du Père), 3 (la mission du Fils), 4 (l'Esprit qui sanctifie l'Église) qui se conclut par une référence à Cyprien («Ainsi l'Église universelle apparait comme "un peuple messianique qui tire son unité de l'unité du Père et du Fils et de l'Esprit-Saint»); auquel correspond le prologue trinitaire du décret Ad Gentes, $\mathrm{n}^{\text {os }} 2$ (le dessein du Père), 3 (la mission du Fils), 4 (la mission du Saint-Esprit).
} 
Ainsi, si Lumen Gentium et, plus largement, le concile Vatican II, n'ont pas fourni un exposé ex professo du rôle de l'Esprit dans l'Église, il faut souligner que toutes les fois qu'on présente l'Église locale (LG, n. 26; CD, n. 11; UR, n. 15), il y est fait mention de l'Esprit-Saint. Ce peuple messianique est convoqué par Dieu, dans l'Esprit-Saint. ${ }^{22}$ C'est donc l'Esprit de Pentecôte qui est au « principe de leur (des chrétiens) rassemblement » (LG, n. 13), l'Église ayant été " manifestée grâce à l'effusion de l'Esprit » (LG, n. 2; UR, n. 2). En ce sens, l'Esprit de Jésus est co-instituant de l'Eglise qui n'apparaît qu'à la Pentecôte ${ }^{23}$. À l'origine et au fondement de la venue des chrétiens en assemblée ${ }^{24}, l^{\prime}$ Esprit n'agit toutefois pas seulement au commencement de l'Église. Il la garde et la maintient en permanence dans la vérité (LG, n. 4) tout au long de son pèlerinage terrestre, et il assure l'unité ceux qu'il a rassemblés. Fondée dans l'Esprit-Saint (GS 42, n. 3), c'est encore grâce à son action puissante que grandit l'unité de l'Église (GS 92, n. 3), puisque c'est l'Esprit qui sans cesse « conserve et fait progresser le troupeau entier du Christ dans l'unité de la foi » (LG, n. 25) C'est dire que l'Esprit est au principe du lien de communion qui existe dans l'Église. Cette unité, il la réalise entre les fidèles (communio fidelium) et entre les Églises (communio ecclesiarum ${ }^{25}$. C'est le même Esprit qui réalise « cette admirable communion des fidèles » $(\mathrm{OE}, \mathrm{n} .2)$ et la communion des Églises locales entre elles ${ }^{26}$ (LG, n. 13; OE, n. 2). Bien plus, c'est « sous le souffle de la grâce de l'Esprit-Saint » que beaucoup d'efforts tendent vers l'unité des Églises séparées (UR, n. 4) et, un jour, c'est dans l'unité de cet Esprit que l'humanité entière sera récapitulée sous un seul chef, le Christ (LG, n. 13).

\footnotetext{
${ }^{22}$ Cf. LG, n. 26 : « Elles sont en effet, chacune sur son territoire, le peuple nouveau appelé par Dieu dans l'Esprit-Saint... »

${ }^{23}$ LG, n. 48 indique bien que l'Esprit est co-instituant de l'Église : "Le Christ élevé de terre a tiré à lui (cf. Jn 12,32); ressuscité des morts (Rm 6, 9), il a envoyé sur ses apôtres son Esprit de vie et par lui a constitué son corps qui est l'Église...» L'Église du Christ est donc aussi celle du Saint-Esprit. Yves Congar a beaucoup développé cette notion de co-institution de l'Église. On trouve des affirmations en ce sens dans plusieurs de ses écrits, notamment dans un chapitre du volume II de son ouvrage Je crois en l'Esprit Saint, "L'Église est faite par l'Esprit. Il en est co-institutant». Sa formulation la plus achevée se trouve sans doute dans un article plus tardif, "Pneumatologie dogmatique " : "Mieux : l'Église, même en ses origines comme institution et société, est faite par deux <missions 》, celle du Fils-Verbe et celle de l'Esprit-Souffle. L'Esprit est * co-instituant > en un sens plus large que nous l'avons dit dans ES [Je crois en l'Esprit Saint] (CONGAR, 1979, T. II, p. 13-24). Avec bien des théologiens aujourd'hui, nous reconnaissons que Jésus avait posé des fondements, mais que la pleine institution de l'Église a été le fait des apôtres après la Pentecôte » (CONGAR, 1982). On verra également l'étude de Sang-Moun (2003).

${ }^{24}$ Cf. aussi GS, n. 40 § 2 et AG, n. 15.

${ }^{25} \mathrm{Il}$ ne faut pas négliger la dimension verticale de la communion, la communion avec Dieu. L'Esprit-Saint, celui-là même qui nous donne un accès direct à Dieu et nous fait communier à sa vie, est encore celui qui établit la communion entre nous. Cette double communion ou, mieux, ce double aspect de la communion est l'œuvre de l'Esprit. Elle est mentionnée en LG, n. 4 et UR, n. 15.

${ }^{26}$ Enfin, selon OE, n. 2, c'est lui qui unit les fidèles au Christ.
} 


\subsection{L'Esprit, source de la diversité et principe d'unité}

L'unité qu'il réalise n'est toutefois pas uniformité. En effet, principe d'unité, l'Esprit est aussi, dans l'Église, principe de distinction et de diversité. C'est dans la communion et le service mutuel que sont unifiés les dons multiples et variés dans l'Église (LG, n. 4; AG, n. 4.). L'Esprit aux souffles variés suscite et promeut la diversité : «Unique est l'Esprit qui distribue ses dons variés pour le bien de l'Église, à la mesure de ses richesses et des exigences des services ${ }^{27} »(L G, n .7)$. Comme au jour de la création, la surabondance de la vie qui jaillit de Dieu se présente dans une grande variété et une multiplicité sans nombre $(\mathrm{Gn} 1,11-25)^{28}$. Ainsi, Dieu ne semble pas craindre le nombre et le divers. Sa grâce est " multiforme » comme le dit l'apôtre Pierre (1 P 4,10). Ce foisonnement d'espèces d'êtres vivants qui grouillent sur la terre, remuent dans les eaux et s'ébattent dans le ciel font sa gloire. Au commencement, était le multiple et la diversité des êtres, et si le salut assume la création pour la renouveler et la mener à son accomplissement, il ne peut s'en saisir que dans sa diversité et sa variété.

La diversité est particulièrement mise en évidence dans l'exposé sur les charismes et le traitement de la question des ministères et des diverses vocations ${ }^{29}$. Toutes sont fondées dans la grâce multiforme et les charismes donnés par l'Esprit Saint. Cette mise en valeur des charismes, nous la trouvons d'abord dans le chapitre deuxième de la Constitution sur le peuple de Dieu. Il s'agit d'un chapitre programmatique et, à l'évidence, il joue un rôle principiel. Ses 22 références à l'Esprit Saint (sur les 109 qu'en compte l'ensemble de la Constitution) se répercutent ensuite dans le chapitre, trois sur la constitution hiérarchique de l'Église (sur les ministères ordonnés), de même qu'au chapitre quatre sur les fidèles laïcs, au chapitre V sur l'appel de tous à la sainteté et au chapitre VI sur la vie religieuse. Cet enseignement sera par la suite repris dans les divers décrets relatifs au ministère de évêques (Christus Dominus), sur le ministère et la vie des prêtres (Presbyterorum ordinis) et sur la rénovation de la vie religieuse (Perfectae caritate). Il est donc devenu impossible, à la suite de Vatican II, d'élaborer une théologie du ministère en procédant à partir du seul rapport Christ-ministre. On est sorti du « christomonisme », pour reprendre la terminologie de Congar. Ce dépassement du christomonisme qui avait caractérisé pendant des siècles la théologie du ministère dans

\footnotetext{
${ }^{27}$ Sur la diversité de ces dons on consultera encore LG 4, 39 : «sous toutes sortes de formes»; AA, n. 3.

${ }^{28}$ On pourrait aussi voir le récit du déluge où toutes les espèces dans leur variété sont sauvées. Gn 7, 20 et 8, 18.

${ }^{29}$ Selon G. Hasenhüttl, les charismes sont le principe même d'organisation de l'Église. Selon sa thèse - que Congar nuance - ce n'est qu'avec la deuxième génération chrétienne que les charismes sont réduits à un seul - celui de vrais enseignements, et attribués aux ministres ordonnés - l'institution. Voir Hasenhüttl (1970a; 1970b).
} 
l'Église catholique est un acquis important de Vatican II qui pose l'Esprit Saint à la source de toute théologie des ministères et de la participation active des fidèles à la vie de l'Église.

\subsection{L'Esprit, fondement du sacerdoce commun}

Le rôle de l'Esprit est particulièrement mis en lumière à un autre endroit stratégique de Lumen Gentium. Il s'agit de son enseignement sur l'exercice du sacerdoce commun des fidèles dans les différents domaines de la vie ecclésiale. Après une introduction générale sur le sacerdoce commun (n. 10), qui comporte une mention de l'Esprit Saint, le texte poursuit par un développement sur l'exercice de ce sacerdoce dans la liturgie ou dans le domaine du munus sanctificandi (n. 11), où l'on trouve deux mentions de l'Esprit Saint, avant de présenter un développement sur sensus fidei ou l'exercice du sacerdoce commun dans le domaine du munus docendi ou de la fonction prophétique de l'Église. Ces développements, en ecclésiologie catholique, représentent une ouverture inattendue. Deux passages de Lumen Gentium, les n. ${ }^{\text {os }} 12$ et 25 abordent la question du sensus fidei et le n. 12 ne compte pas moins de quatre références à l'Esprit Saint.

Le Peuple saint de Dieu a part également à la fonction prophétique du Christ, en rendant un vivant témoignage à son endroit, avant tout par une vie de foi et de charité et en offrant à Dieu un sacrifice de louange, c'est-à-dire le fruit de lèvres qui ont reçu l'onction du Saint (1 Jn 2,20 et 27) ne peut pas errer dans la foi; et il manifeste cette prérogative au moyen du sens surnaturel de la foi commun à tout le peuple, lorsque " depuis les évêques jusqu'au dernier des fidèles laïcs ", il fait entendre son accord universel dans les domaines de la foi et de la morale. C'est, en effet, dans ce sens de la foi éveillé et nourri par l'Esprit de vérité que le Peuple de Dieu, fidèlement soumis à la conduite du magistère sacré, accueille vraiment non pas une parole humaine mais la parole de Dieu (1 Th 2,13), qu'il adhère indéfectiblement « à la foi qui fut une fois pour toutes transmise aux saints » (Jude 3), qu'il approfondit correctement cette même foi et la met plus pleinement en œuvre.

On constate donc qu'on n'a pas fait que « saupoudrer » un peu d'Esprit Saint dans les textes conciliaires, mais que sa mention s'insère à des moments déterminants de son enseignement.

\section{D'autres domaines cruciaux}

Les discussions postconciliaires sur la pneumatologie du concile se sont concentrées dans le domaine de l'ecclésiologie. Cet ecclésiocentrisme laisse cependant dans l'ombre un pan important de la pneumatologie du concile. En effet, si l'Esprit n'est pas assigné à résidence dans l'Église, celui-ci agit dans le monde ou hors des frontières de l'Église. À ce chapitre, il importe de 
ne pas se contenter de lire la constitution Lumen Gentium et tous les décrets ecclésiologiques qui s'y rattachent, mais d'élargir la lecture à la Constitution Gaudium et Spes, au Décret sur l'activité missionnaire de l'Église et à la Déclaration sur les religions non chrétiennes. En somme, il faut lire tout ce qui concerne ces espaces situés hors des frontières de l'Église. C'est là que nous retrouvons des éléments importants de la pneumatologie de Vatican II.

Peu après le concile, Congar rapporte ce propos : «Tel observateur disait : ce n'est pas parce que le Saint-Esprit sera mentionné trente fois, soixante-dix fois (ainsi Gaudium et Spes), qu'il y aurait vraiment " pneumatologie » (CONGAR, 1973, p. 121). Cela est vrai, certes. Toutefois, encore une fois, l'approche statistique n'est pas la meilleure voie pour une telle recherche. Il importe donc de reprendre la lecture de Gaudium et spes en y recherchant le rôle que la constitution accorde à l'Esprit Saint et, cette fois, pas d'abord dans l'Église, mais dans le monde. L'énoncé principiel se retrouve au $\mathrm{n}^{\circ} 10$ où l'on tourne son regard, paraphrasant le premier chapitre du livre de la Sagesse, vers « l'Esprit du Seigneur qui remplit l'univers ». Ce numéro, qui conclut l'exposé préliminaire sur les signes des temps et qui en reprend la notion, est intitulé, de manière significative "Répondre aux appels de l'Esprit ». Dans les numéros 4 à 10, l'Église s'est intéressée aux espoirs et aux angoisses des hommes de ce temps, a considéré les mutations profondes du monde, les changements dans l'ordre social, mais aussi les changements dans l'ordre moral et religieux, présenté ses déséquilibres et a identifié les aspirations et les interrogations profondes du genre humain. Bref, elle s'est mise à l'écoute de ce qui est à l'œuvre dans le monde.

C'est cette attitude empathique d'écoute et de regard bienveillant qui la conduit à découvrir l'Esprit à l'œuvre dans le monde. Elle découvre ainsi que « l'Esprit de Dieu, qui par une providence admirable, conduit le cours des temps et rénove la face de la terre, est présent à cette évolution [de l'ordre social] »(GS, n. 26,4.). En somme, la reconnaissance des semences du Verbe dans la vie du monde, de la grâce de Dieu qui agit au cœur de l'humanité ou de l'Esprit qui remplit l'univers est la trame de fond de la fresque si neuve que représente Gaudium et Spes. En tête de la Constitution, il y a cette affirmation que l'Esprit remplit l'univers,

Cet énoncé principiel commande tous les autres développements importants sur l'Esprit Saint agissant au cœur du monde et de l'humanité que l'on retrouve dans la Constitution. Il ne s'agit pas d'un développement systématique, mais d'une construction, par touches successives, qui donne son caractère au tableau d'ensemble. Ainsi au $n^{\circ} 22,5$ autre moment charnière du texte puisqu'il conclut le chapitre premier sur la dignité de la personne humaine, on reprend le thème de l'action de l'Esprit, bien au-delà des frontières de l'Église :

Et cela ne vaut pas seulement pour ceux qui croient au Christ, mais bien pour tous les hommes de bonne volonté, dans le cœur desquels, invisiblement, agit 
la grâce. En effet, puisque le Christ est mort pour tous et que la vocation dernière de l'homme est réellement unique, à savoir divine, nous devons tenir que l'Esprit-Saint offre à tous, d'une façon que Dieu connaît, la possibilité d'être associé au mystère pascal.

On revient sur le même thème, au chapitre II [n. 26, 4], affirmant que «L'Esprit de Dieu qui, par une providence admirable, conduit le cours des temps et rénove la face de la terre, est présent à cette évolution [de la communauté humaine]. » Enfin, la finale du chapitre III sur l'activité humaine dans l'univers reprend la même idée au n. 38 :

le Christ, à qui tout pouvoir a été donné, au ciel et sur la terre, agit désormais dans le cœur des hommes par la puissance de Son Esprit; Il n'y suscite pas seulement le désir du siècle à venir, mais par là même anime aussi, purifie et fortifie ces aspirations généreuses qui poussent la famille humaine à améliorer ses conditions de vie et à soumettre à cette fin la terre entière.

Le chapitre IV ne fait pas exception. L'Église ne se présente pas de manière autocentrée. Elle reconnaît à nouveau le rôle de l'Esprit dans le monde : «Elle sait aussi que l'homme, sans cesse sollicité par l’Esprit de Dieu, ne sera jamais tout à fait indifférent au problème religieux. » (GS, n. 41, 1). Ainsi, aucun chapitre de la première partie de la Constitution ne fait exception. Le thème de la présence de l'Esprit (à reconnaître et qui devance l’Eglise) est récurrent.

Cet enseignement devait naturellement connaître des répliques dans le Décret sur l'activité missionnaire de l'Église. En effet, la réflexion sur l'activité missionnaire de l'Église intègre le fait que l'Esprit devance la prédication de l'Église, notamment à travers les semences du $\operatorname{Verbe}^{30}$.

De plus, si Lumen Gentium reconnaissait que l'Esprit était donné aux chrétiens non catholiques ${ }^{31}$, ce qui constituait une avancée œcuménique remarquable, il ne reconnaissait pas que l'Esprit était donné aux non chrétiens $^{32}$. Il faudra attendre le Décret Ad Gentes pour que l'on reconnaisse

\footnotetext{
${ }^{30}$ Voir notamment Ad Gentes, n. 15 : «Quand l'Esprit-Saint, qui appelle tous les hommes au Christ par les semences du Verbe et la prédication de l'Évangile et produit dans les cœurs la soumission de la foi ». On verra aussi le n. 13, qui affirme clairement que c'est l'Esprit qui ouvre les cœurs des païens, rendant opérante la parole proclamée par le missionnaire.

${ }^{31}$ Voir LG, n. 15 : « À cela s'ajoute la communion par la prière et d'autres bienfaits spirituels; et même une union réelle dans l'Esprit-Saint, car l'Esprit agit également en eux par ses dons et ses grâces, avec sa puissance sanctificatrice; et il a donné à certains d'entre eux une vertu qui les a fortifiés jusqu'à l'effusion de leur sang. Ainsi l'Esprit éveille-t-il en tous les disciples du Christ le désir et oriente-t-il leur activité afin que tous s'unissent pacifiquement, de la manière que le Christ a fixée, en un seul troupeau et sous un seul Pasteur. » Cet enseignement est repris en Unitatis Redintegratio au n. 3.

${ }^{32}$ Le développement du n. 16 (les non-chrétiens), à la différence du n 15 (les chrétiens non catholiques), ne mentionne pas l'Esprit Saint. Cette différence est également marquée dans le fait que UR considère les communautés séparées comme des moyens de salut, ce que l'on ne dit pas à propos des religions non chrétiennes : "L'Esprit du Christ, en effet, ne refuse pas de se servir d'elles comme de moyens de salut dont la force dérive de la plénitude de grâce et de vérité qui a été confiée à l'Église catholique. » (UR, n. 3) On verra aussi le $n^{\circ} 4$
} 
explicitement que les membres du peuple juif, tout au moins, avaient reçu l'Esprit Saint : "Sans l'ombre d'un doute, le Saint-Esprit était déjà à l'œuvre avant la glorification du Christ (n. 4) ${ }^{33}$. » On regrettera cependant que la Déclaration sur les religions non chrétiennes ne construise pas sur cet élément et ne table pas sur l'énoncé de Gaudium et Spes, suivant lequel " l'Esprit-Saint offre à tous, d'une façon que Dieu connaît, la possibilité d'être associé au mystère pascal » (n. 22).

\section{Conclusion}

Le concile Vatican II n'arrive certainement pas à un déploiement complet de la pneumatologie (en ecclésiologie) - nous l'avons bien montré en en indiquant les limites relevées par les observateurs, bien qu'il soit injuste de dire qu'il n'a fait que "saupoudrer » un peu d'Esprit Saint dans des développements dont le principe n'est que christologique ${ }^{34}$. Il faut donc, d'une part, reconnaître les limites de la pneumatologie de Vatican $\mathrm{II}^{35}$, reconnaître ensuite les progrès importants qu'il opère en ce domaine dans l'enseignement de l'Église catholique, progrès que reconnaissent $\mathrm{du}$ reste les observateurs non catholiques ${ }^{36}$, reconnaître enfin qu'il pose

qui affirme, autrement, que l'Esprit est à l'œuvre en dehors de l'Église catholique : «Il ne faut pas non plus oublier que tout ce qui est accompli par la grâce de l'Esprit-Saint dans nos frères séparés peut contribuer à notre édification ».

${ }^{33}$ En note, on fondera de la manière suivante cette affirmation : " C'est l'Esprit-Saint qui a parlé par les prophètes : Symb. de Constantinople (Denz. 150 [86]); S. Léon le Grand, Sermon 76 (P.L. 54, 405-406) : " Quand au jour de la Pentecôte l'Esprit-Saint remplit les disciples du Seigneur, ce ne fut pas le début d'un don, mais une largesse surajoutée à d'autres: les patriarches, les prophètes, les prêtres, tous les saints qui vécurent aux temps anciens ont été nourris du même Esprit sanctifiant... bien que la mesure des dons ait été différente «. De même le Sermon 77, I (P.L. 54, 412); Léon XIII, Encycl. Divinum illud, 9 mai 1897 (AAS 1897, 650-651). De même S. Jean Chrysostome, bien qu'il insiste sur la nouveauté de la mission du Saint-Esprit au jour de la Pentecôte: In Eph., c. 4, Hom. 10, 1 (P.G. 62, 75). »

${ }^{34}$ Congar notait déjà (CONGAR, 1973, p. 121-122) : un théologien protestant, G. Westphal, a pu écrire que l'effort entrepris avait seulement abouti à " saupoudrer " les textes de Saint-Esprit. Je crois que cela est injuste, on pourrait en faire la preuve. » Bien qu'il trouve exagérée les critiques de Nissiotis et Lossky (« Nous croyons ces critiques exagérées et insuffisamment fondées. Ni au point de vue biblique ni au point de vue dogmatique on ne peut poser une économie du Paraclet qui serait autonome par rapport à l'économie du Verbe incarné. »(CONGAR, 1970, p. 42)), il s'emploiera cependant à relever ce qu'il y a de juste dans ces critiques qui l'ont aiguillonné à reprendre la question de la pneumatologie en ecclésiologie catholique.

${ }^{35}$ Congar reconnaissait volontiers les limites de la pneumatologie du concile : " Nous reconnaissons que Vatican II est, en bien des domaines, imparfait. Beaucoup de ses vues sont, sinon des compromis, du moins des ébauches et se tiennent, en quelque sorte, à mi-chemin » (CONGAR, 1981, p. 130).

${ }^{36}$ Voir note 8 . Les sévères critiques de Nissiotis, qui portaient sur le schéma et non sur le texte définitif, sont cependant tempérées par J. Zizioulas. Lui-même reconnaissait, comme on l'a montré, que la révision du schéma l'avait beaucoup amélioré. 
des bases sur lesquelles la théologie catholique pourra construire par la suite. C'est sur ce dernier point que je voudrais conclure, après avoir mis en évidence, sur la base des textes du concile, la pneumatologie de Vatican II. Certes, cette présentation appelle un complément, l'examen de la réception de cette pneumatologie, non seulement dans les textes magistériels et théologiques, mais également dans la vie de l'Église, sa pratique, sa spiritualité et sa liturgie. Cela n'est pas le but poursuivi par cet article, mais nous pouvons au moins en dire brièvement un mot par manière d'ouverture.

Tous les développements postconciliaires dans le domaine de la participation de tous les fidèles à la vie de l'Église et, singulièrement, dans celui sur la théologie des ministères, spécialement des ministères exercés par les fidèles laïcs, est tributaire de l'enseignement de Vatican II sur l'Esprit Saint et sur les charismes octroyés à tous les membres du Christ. Nous n'en serions pas là aujourd'hui si Vatican II n'avait pas posé les fondements. Le dépassement du christomonisme nous a fait passer d'un modèle ministériel dans lequel le prêtre, représentant du Christ, agissait au nom, en faveur et pour les fidèles qui étaient bénéficiaires de ses soins et de ses actions à une Église où tous les fidèles, membres actifs du Corps du Christ, sont sujets de droits et d'initiatives. Cela est vrai dans l'action liturgique, certes, mais l'est également dans les domaines de la fonction prophétique et du gouvernement ecclésial. Sans les perspectives pneumatologiques développées par Vatican II, nous n'aurions pas aujourd'hui les développements que nous connaissons sur le sensus fidei fidelium $^{37}$ et sur la synodalité, deux thèmes qui traversent le magistère du pape François.

Si Vatican II n'avait pas posé les bases de la réflexion sur l'activité de l'Esprit dans le monde, la réflexion missiologique n'aurait pas connu les développements qu'elle a connu depuis le concile, pas plus que la théologie des religions. À titre d'exemple, on n'aurait pas les beaux paragraphes de

\footnotetext{
${ }^{37}$ Les références à l'Esprit Saint sont constantes, depuis Vatican II jusqu'à Evangelii gaudium, lorsqu'il s'agit d'aborder la question du sensus fidei. La question du sensus fidei fidelium manifesté en particulier chez les gens simples est constante chez le pape François. On peut rappeler ce passage de son premier angelus sur l'écoute de la foi vécue des pauvres et des petits. Il évoque alors l'instinct de la foi ou le flair spirituel d'une vieille dame : "Est venue à moi une femme âgée, humble, très humble, elle avait plus de quatre-vingts ans. Je l'ai regardée et je lui ai dit : "Grand-mère [...] vous voulez vous confesser ? ». " Oui ! », m'a-t-elle dit. « Mais si vous n'avez pas péché... ». Et elle m’a dit : « Nous avons tous péché... ! ». " Mais peut-être le Seigneur ne les pardonne pas... ». « Le Seigneur pardonne tout ! », m'a-t-elle dit : sûre d'elle. " Mais comment le savez-vous, vous, Madame? ». "Si le Seigneur ne pardonnait pas tout, le monde n'existerait pas ». Il m'est venue l'envie de lui demander : «Dites-moi, Madame, vous avez étudié à la Grégorienne ? ", parce que cela est la sagesse que donne l'Esprit Saint ; la sagesse intérieure vers la miséricorde de Dieu. » (Angélus, 17 mars 2013) Il reprendra la question dans son entretien avec son confrère Spadaro. Voir aussi Evangelii gaudium aux $\mathrm{n}^{\text {os }} 119,124-126$ et 198.
} 
l'encyclique Redemptoris Missio sur la présence et l'action de l’Esprit Saint en dehors des frontières de l'Église.

28. L'Esprit se manifeste d'une manière particulière dans l'Église et dans ses membres; cependant sa présence et son action sont universelles, sans limites d'espace ou de temps. Le Concile Vatican II rappelle l'œuvre de l'Esprit dans le cœur de tout homme, par les "semences du Verbe ", dans les actions même religieuses, dans les efforts de l'activité humaine qui tendent vers la vérité, vers le bien, vers Dieu.

[...] « nous devons tenir que l'Esprit Saint offre à tous, d'une façon que Dieu connaît, la possibilité d'être associés au Mystère pascal ». Dans tous les cas, l'Église sait que «l'homme, sans cesse sollicité par l'Esprit de Dieu, ne sera jamais tout à fait indifférent au problème religieux » et qu'il "voudra toujours connaître, ne serait-ce que confusément, la signification de sa vie, de ses activités et de sa mort ». L'Esprit est donc à l'origine même de l'interrogation existentielle et religieuse de l'homme qui ne naît pas seulement de conditions contingentes mais aussi de la structure même de son être.

La présence et l'activité de l'Esprit ne concernent pas seulement les individus, mais la société et l'histoire, les peuples, les cultures, les religions. En effet, l'Esprit se trouve à l'origine des idéaux nobles et des initiatives bonnes de l'humanité en marche: "Par une providence admirable, il conduit le cours des temps et rénove la face de la terre ». Le Christ ressuscité " agit désormais dans le cœur des hommes par la puissance de son Esprit; il n'y suscite pas seulement le désir du siècle à venir, mais, par là même, anime aussi, purifie et fortifie ces aspirations généreuses qui poussent la famille humaine à améliorer ses conditions de vie et à soumettre à cette fin la terre entière ». C'est encore l'Esprit qui répand les "semences du Verbe », présentes dans les rites et les cultures, et les prépare à leur maturation dans le Christ.

29. Ainsi l'Esprit, qui « souffle où il veut » $(J n 3,8)$ et qui « était déjà à l'œuvre avant la glorification du Christ ", lui qui "remplit le monde et qui, tenant unies toutes choses, a connaissance de chaque mot » $(S g 1,7)$, nous invite à élargir notre regard pour contempler son action présente en tout temps et en tout lieu. Moi-même, j'ai souvent renouvelé cette invitation et cela m’a guidé dans mes rencontres avec les peuples les plus divers. Les rapports de l'Église avec les autres religions sont inspirés par un double respect: "Respect pour l'homme dans sa quête de réponses aux questions les plus profondes de sa vie, et respect pour l'action de l'Esprit dans l'homme ». La rencontre inter-religieuse d'Assise, si l'on écarte toute interprétation équivoque, a été l'occasion de redire ma conviction que « toute prière authentique est suscitée par l'Esprit Saint, qui est mystérieusement présent dans le cœur de tout homme ».

$[\ldots]$

L'action universelle de l'Esprit n'est pas à séparer de l'action particulière qu'il mène dans le corps du Christ qu'est l'Église. En effet, c'est toujours l'Esprit qui agit quand il vivifie l'Eglise et la pousse à annoncer le Christ, ou quand il répand et fait croître ses dons en tous les hommes et en tous les peuples, amenant l'Église à les découvrir, à les promouvoir et à les recevoir par le dialogue. Il faut accueillir toutes les formes de la présence de l'Esprit avec respect et reconnaissance, mais le discernement revient à l'Église à laquelle le Christ a donné son Esprit pour la mener vers la vérité tout entière $(\ln 16,13)$. 


\section{Sigles}

$\mathrm{AD}=$ Acta et Documenta
$\mathrm{AG}=$ Décret Ad Gentes
$\mathrm{AS}=$ Acta Synodalia
$\mathrm{CD}=$ Décret Christus Dominus
$\mathrm{GS}=$ Constitution Pastorale Gaudium et Spes
$\mathrm{LG}=$ Constitution Dogmatique Lumen Gentium
$\mathrm{OE}=$ Décret Orientalium Ecclesiarum
$\mathrm{UR}=$ Décret Unitatis Redintegratio

\section{References}

ACTA et documenta Concilio oecumenico Vaticano II apparando [AD]. Series I (Antepreparatoria): Acta S. Pontificis Ioannis XXIII. Vatican: Typis polyglottis vaticani, 1960. v. 1.

ALBERIGO, G.; MAGISTRETTI, F. Constitutionis Dogmaticae Lumen Gentium. Synopsis Historica. Bologna: Istituto per la Scienze religiose, 1975.

ATHANASE. Apologia secunda.

BOUYER, L. L'Église de Dieu. Corps du Christ et temple de l'Esprit. Paris: Cerf, 1970.

CAZELLE, H. Le Saint-Esprit dans les textes de Vatican II. In: CAZELLE, H.; EVDOKIMOV, P.; GREINER, A. Le mystère de l'Esprit-Saint. Paris: Mame, 1968. p. 161-186.

CHARRUE, A. M. Le Saint-Esprit dans «Lumen Gentium». Ephemerides Theologicae Lovanienses, Louvain, v. 45, n. 3, 358-379, 1969.

CLÉMENT, O. Quelques remarques d'un orthodoxe sur la constitution «de Ecclesia». OEcumenica: Jahrbuch für ökumenische Forschung, Strasbourg, v. 1, p. 97-116, 1966.

CONGAR, Y.; KÜNG, H.; O’HANLON, D. Discours au concile Vatican II. Paris: Cerf, 1964.

CONGAR, Y. La pneumatologie dans la théologie catholique. Revue des Sciences Philosophiques et Théologiques, Paris, v. 51, n. 2, p. 250-258, 1967.

CONGAR, Y. Pneumatologie ou «Christomonisme» dans la tradition latine? In: COPPENS, J. (Dir.). Ecclesia a Spiritu Sancto edocta. Mélanges théologiques. Hommage à Mgr Gérard Philips. Gembloux: J. Duculot, 1970. p. 41-63.

CONGAR, Y. Actualité d'une pneumatologie. Proche-Orient chrétien, Beirute, v. 23, p. 121-132, 1973.

CONGAR, Y. Je crois en l'Esprit Saint. Paris: Cerf, 1979. 2 v.

CONGAR, Y. Les implications christologiques et pneumatologiques de l'ecclésiologie de Vatican II. In : ALBERIGO, G. (Dir.). Les Églises après Vatican II: Dynamisme et prospective. Paris: Beauchesne, 1981. p. 117-130. 
CONGAR, Y. Pneumatologie dogmatique. In: LAURENT, B.; REFOULÉ, F. (Dir.). Initiation à la pratique de la théologie. Dogmatique 1. Paris: Cerf, 1982. v. 2, p. 483-516.

CONGAR, Y. L'Église en cartes: À propos d'un traitement informatique des textes de Vatican I. In: CONGAR, Y. Le concile de Vatican II. Son Église peuple de Dieu et corps du Christ. Paris: Beauchesne, 1984. p. 73-78

FESQUET, H. Le Journal du concile. Paris: Robert Morel, 1966.

FRANÇOIS, Pape. Angélus. Roma: 17 mar. 2013.

GIL HELLÍN, G. Concilii Vaticani II synopsis in ordinem redigens schemata cum relationibus necnon patrum orationes atque animadversiones: Constitutio dogmatica de Ecclesia. Città del Vaticano: Libreria Editrice Vaticana, 1995.

GIL HELLÍN, G. Concilii Vaticani II synopsis in ordinem redigens schemata cum relationibus necnon patrum orationes atque animadversiones: Decretum de Presbyterorum Ordinis. Città del Vaticano: Libreria editrice Vaticana, 1996.

HASENHÜTTL, G. Charisma Ordnungsprinzip der Kirche. Freiburg im Breisgau: Herder, 1970a.

HASENHÜTTL, G. Les charismes dans la vie de l'Église. In: CONGAR, Y. Vatican II. L'Apostolat des laïcs. Paris: Cerf, 1970b. p. 203-214.

JEAN XXIII, Pape. Motu proprio Superno Dei nutu. Roma: 05 jui. 1960.

NISSIOTIS, N. K. The main Ecclesiological Problem on the Second Vatican Council and the Position of the Non-Roman Churches facing it. Journal of Ecumenical Studies, Philadelphia, v. 2, n. 1 p. 31-62, 1965.

NISSIOTIS, N. K. Comité Central du Conseil œcuménique, Genève, 8-17 février 1966 : Rapports sur le 2e Concile du Vatican. Istina, Boulogne-Billancourt, v. 11, n. 2-3, p. 249-252, 1966.

NISSIOTIS, N. K. La pneumatologie ecclésiologique au service de l'unité de l'Église. Istina, Boulogne-Billancourt, v. 12, p. 323-340, 1967.

NISIUS, A. L'Esprit Saint et l'Église dans l'œuvre d'Yves Congar. Transversalités, Paris, n. 98, p. 109-155, 2006.

PAUL VI, Pape. Audience Générale. Roma: 06 jui. 1973.

PHILIPS, G. L'Église et son mystère au II concile du Vatican. Paris: Desclée, 1967. 2 v. ROUX, A. Le décret sur l'activité missionnaire de l'Église. In: MARTIN, J.-M. (Dir.). Vatican II. Points de vue de théologiens protestants. Paris: Cerf, 1967. p. 109-127.

SANG-MOUN, K. Le Christ et l'Esprit Saint «co-instituants» de l'Église. Étude théologique et incidences pastorales de la christologie pneumatologique de Y. Congar. 2003. Mémoire de maîtrise, Institut Catholique de Paris, Paris, 2003.

TONIOLO, E. La Beata Maria Vergine nel Concilio Vaticano II: Cronistoria del Capitolo VIII della Costituzione dogmatica 'Lumen Gentium' e Sinossi di tutte le redazioni. Roma: Centro di Cultura Mariana « Madre della Chiesa », 2004.

VAJTA, V. "Renewal of Worship": De sacra Liturgia. In: LINDBECK, G. (Ed.). Dialogue on the Way. Minneapolis: Augsburg, 1965. p. 101-128. 
VISCHER, L. L'Église, communauté de l'Esprit: réflexions sur la seconde session du concile du Vatican. Lumière et vie, Lyon, v. 13, n. 67, p. 25-46, 1964.

ZIZIOULAS, J. Christologie, pneumatologie et institutions ecclésiales. In: ALBERIGO, G. (Dir.). Les Églises après Vatican II. Dynamisme et prospective. Paris: Beauchesne, 1981. p. 131-148.

WASSELYNCK, R. Les prêtres. Élaboration du décret de Vatican II. Histoire et genèse des textes conciliaires. Paris: Desclée \& Cie, 1968.

WESTPHAL, G. Vie et foi du Protestant. Paris: Centurion, 1966.

Article soumis à l'evaluation le 23.04.2020 et approuvé le 14.08.2020.

Gilles Routhier, est Docteur en Théologie (Institut catholique de Paris) et Docteur en Anthropologie Religieuses et Histoire des Religions (Université de Paris IV - Sorbonne), Doyen de la Faculté de théologie et de sciences religieuses, Université Laval. Orcid.org/0000-00021926-5813. Email: gilles.routhier@ftsr.ulaval.ca

Adresse: Pavillon Félix-Antoine-Savard, local 730

2325, rue des Bibliothèques - Université Laval

Québec - G1V 0A6 - Canadá 